\title{
Using constraints to address the instabilities of automated prestack velocity analysis
}

\author{
Christof Stork* and Robert W. Clayton $¥$
}

\begin{abstract}
Generalized prestack velocity analysis methods that use an automated approach to resolve laterally variable interval velocity fields are beset by a series of problems. The problem of resolving lateral velocity variations has inherent complications that prevent automated methods from being robust enough to be applied routinely to data from a variety of geologic provinces. The use of automated prestack velocity analysis methods will not eliminate the step of carefully producing an initial velocity model derived from regional geologic information and an interpretation of a conventionally processed section. For the methods to regularly produce useful additional information, the unique characteristics of each application must be input into the prestack velocity analysis with the use of inversion constraints. These constraints serve either to adapt the generalized prestack velocity analysis to a focused objective in a particular area or to provide iterative, interpretational tools that help the user produce a velocity model.
\end{abstract}

\section{INTRODUCTION}

Accurate knowledge of the broad components of seismic velocities (those components that control transmission of seismic energy) is essential for transforming surface reflection time data into depth images of reflector locations. Advanced prestack migration schemes exist that could produce images in many structurally complex areas as clear as those produced in simpler areas if accurate velocities were attainable. Present routine methods of velocity determination, however, are generally unable to handle velocity variations commonly found in a variety of geologic settings. Causes of such velocity variations include lithologic changes, permafrost variations, gas pockets, basin thinning, salt layers with varying thickness, fault blocks, and dynamic statics. The accurate determination of laterally varying velocities is a key step for improving seismic data processing in these areas.

Although the same velocity field affects reflection and transmission of the wavefield, the components of the velocity field that affect each process are separable (Mora, 1987). Reflections are caused by the small scale, high wavenumber components of the velocity field (interfaces), while transmission is affected by the broad, low wavenumber components. This separation is more distinct for band-limited energy propagating predominantly in one direction, such as vertically, which is the case for recorded seismic data.

The low wavenumber component of the subsurface velocity field may not correlate with reflector locations. Several factors (i.e., variations in the stress field, lithologic changes, and changes of gas saturation, among others) can significantly affect seismic velocity between reflector locations. We do not assume that the resolution of the interfaces predicts the low wavenumber structure of the velocity field. Such an assumption would prevent parameterizing the velocity field as a collection of constant velocity zones between reflector locations. While we consider the low wavenumber component of the velocity field to have no inherent relationship with the reflector positions, such relationships can be imposed optionally as constraints if geologic information in an area warrants it.

Here, we address the viability of generalized prestack velocity analysis. By generalized, we mean that (1) no implicit assumptions about the velocity field are made, (2) the velocity field can take on any shape down to a certain resolution, and (3) any moveout pattern on a CMP gather can be represented. A velocity analysis method that is generalized can be applied to data from any geologic structure.

A variety of generalized velocity analysis techniques has been proposed for using the prestack transmission information for velocity analysis. Those that directly invert un-

Manuscript received by the Editor April 25, 1990; revised manuscript received August 12, 1991

*Formerly Seismological Lab, California Institute of Technology, Pasadena, CA; presently Advance Geophysical, 7409 S. Alton Ct., Suite 100, Englewood, CO 80112 .

†Seismological Lab, California Institute of Technology, Pasadena, CA 91125.

(C) 1992 Society of Exploration Geophysicists. All rights reserved. 
stacked data have been classified as tomography. Bishop et al. (1985), Williamson (1986; 1990), Bording et al. (1987), Kennett et al. (1988), Farra and Madariaga (1988), and Stork and Clayton (1991) propose traveltime inversion. Tarantola (1984) and Mora (1987) propose an iterated Born inversion approach of the waveform data. Thorson (1984) proposes a layer stripping approach using nonhyperbolic stack optimization. Sneider et al. (1988) propose using a Monte-Carlo or a Simplex search algorithm to optimize waveform correlation between data and synthetic seismograms from a model. These tomographic techniques measure the "goodness of fit" of their model by how well it predicts the data.

Another related class of prestack velocity analysis techniques, generally called migration velocity analysis, is based on optimizing the migration of the prestack data. Yilmaz and Chambers (1984), Faye and Jeannot et al. (1986), Deregowski (1990), Fowler (1988), and Etgen (1990) employ semblence methods to measure the degree of similarity between the redundant images of the subsurface. Gardner et al. (1974), MacBain (1989), Julien et al. (1988), verWest (1990), and van Trier (1990) show how a person can quality control the similarity between prestack migration panels. The approach of Sword (1987) can be considered to be a ray-based method analogous to migration velocity analysis that uses the migrated traveltimes for the objective function.

These techniques that use the prestack transmitted information differ from each other in the model parameterization, the data representation, the objective function, and the inversion procedure. But they share the goal of resolving velocity variations with the same data, the information transmitted through the velocity variations using a generalized approach. Here we do not address the questions of the most optimum representation, robust determination, or efficient use of the transmitted information. We use the simplest data, traveltimes, and the simplest energy propagation method, ray tracing, to analyze the characteristics of generalized prestack velocity analysis.

Since seismic reflection data are band-limited, generally propagate in a vertical direction, and generally encounter a subsurface that contains mild horizontal variations (even in complex structure), they undergo mostly nondispersive transmission and precritical reflection. Consequently, ray tracing is an accurate method of simulating waveform propagation. Our results may be applicable to waveform-based inversion methods.

Analytic studies of generalized velocity analysis using traveltimes are performed in Stork (1992), Bube et al. (1985; 1989). Stork (1992) finds that significant aspects of reflector depth are unresolvable while Bube et al. $(1985 ; 1989)$ conclude that most components of reflector depth are resolvable.

Synthetic modeling below and the experience of Farra and Madariaga (1988) using traveltimes and rays demonstrate that generalized prestack velocity analysis is beset with numerous problems inherent to the seismic reflection survey: the lack of horizontal rays, the unavailability of an accurate reference model, and the intrinsic coupling between velocity and reflector. With these intrinsic problems of velocity analysis and the additional problems of 3-D effects, anisotropy, edge effects, and variable data quality, it $\mathrm{is}$ unlikely that generalized prestack velocity analysis without constraints will be effective for many data applications.

Examples below and the experience of van Trier (1990) and Stork (1988) show, however, that prestack velocity analysis is effective if the generalized approach can be customized to a particular application with the use of constraints. These constraints are derived from geologic information available in some individual areas. When adequate geologic information is not available, artifacts from the inherent problems of prestack velocity analysis cannot be avoided initially. However, these artifacts may be identifiable by an explorationist as indicating unreasonable geology. Constraints can be used to attempt to eliminate these artifacts. The flexible application of constraints enables prestack velocity analysis to be an iterative, interpretational technique that helps the user produce a velocity-depth model that is both consistent with the prestack data and geologically reasonable.

\section{SYNTHETIC MODELING}

Velocity analysis is intrinsically coupled with reflector imaging (Stork and Clayton, 1991). One task cannot be done without the other. No formulation has yet been accepted by the geophysical community as being able to properly represent the coupled velocity-reflector depth problem in areas of nonflat geology. The raypath inverse formulation, called reflection tomography, originally presented by Bishop et al. (1984) and also used by Williamson (1986; 1990), Bording et al. (1987), Kennett et al. (1988), and Farra and Madariaga (1988) allows complete and efficient representation of the coupled velocity-reflector depth problem. It serves as the basis for the synthetic modeling performed below.

Ray trace tomography traces rays through a reference model to determine which velocity cells and reflector locations are connected with a given arrival on a trace. The velocity cells and reflector locations along these raypaths are adjusted using matrix inversion so that the predicted traveltimes of a model best match the traveltimes of the arrivals in the data. The amplitude information is not used in our modeling.

Reflection tomography is an inversion problem that, like many in geophysics, has a large number of parameters. Only a small subset of the many possible models can be adequately considered. Since it is unlikely that any of the attempted models will be satisfactory, they are used as a starting point which is adjusted with an iterative gradient inversion method to fit the data. The starting point, called a reference model, is used to compute the raypaths that relate the data to the model. The inversion, which is a linear process, does not take into account that changing the veloeity changes the raypaths. Consequently, errors in the raypaths have an effect on the resulting inversion which is a nonlinear effect. Additional ray tracings and inversions generally reduce these nonlinear effects, but do not entirely remove them.

The implementation used here is described in detail in a companion paper, Stork and Clayton (1991), and in Stork (1988). We find the following aspects important in our implementation: (1) including a reflector term in the inversion; (2) varying the relative weights assigned to velocity and 
reflector depth in the inversion; (3) handling nonuniform illumination using the weights of the Dines and Lytle backprojection method; (4) using migration to image the reflector rather than using the output from the inversion; (5) characterizing the inversion by the eigenvalue range inverted; (6) inverting to small eigenvalue; and (7) including a variety of geologic information with the use of inversion constraints. We feel that the results and conclusions below are applicable to many methods in addition to the one used here.

The synthetic data from a model are computed by ray tracing a model to compute traveltimes. The correct raypaths are determined using a ray-tracing method similar to that of Langan et al. (1984). No noise is introduced into the data. The model used for forward ray tracing and the one used for inversion consisted of $200 \times 35$ sq cells although forced smoothing in the solution increases effective cell size by a factor of 4 (Stork and Clayton, 1991). Unless otherwise noted, the data collection for all models simulates a reflection survey geometry with a cable length of $10500 \mathrm{ft}(3200$ $\mathrm{m})$. This is twice the depth to the reflector and in a constant velocity media produces a maximum ray coverage of \pm 45 degrees from vertical. This ray coverage is greater than that generally obtained which improves resolution. When two or more raypaths exist to a receiver, the one with the largest amplitude is taken. The synthetic data are sufficiently dense that they can be considered to be a continuum compared with the cell size. The eigenvalue range over which the reflection tomography inversion is performed is controlled. Unless otherwise noted, the range inverted is from 1.0 to 0.04 (with a smooth roll off), where the maximum eigenvalue of the inverse problem has been set to 1.0 (Stork and Clayton, 1991). Those components of the model that correspond to eigenvectors with eigenvalue below 0.04 are generally too sensitive to typical noise in the data and will not be inverted. Since the inversions are described by the inverted eigenvalue range, they can be reproduced using other iterative methods and are not unique to the iterative method employed here. The weights imposed by Dines and Lytle's
(1979) back-projection on the model and data space are mild because a uniform coverage exists and raypath lengths do not vary greatly.

\section{Original Model}

Figure 1 presents the physical model that will be used repeatedly for synthetic modeling to demonstrate several characteristics. It contains three blocks at different depths of velocity 25 percent slower than the surrounding velocity. The blocks can represent gas pockets or fault blocks.

The zero offset synthetic seismograms for this model (produced using a 45 degree finite-difference one-way propagator) are shown in Figure 2. Under the assumption that the velocity of the blocks cannot be determined using conventional means, we migrate the data through the constant background velocity field. The result is shown in Figure 3. The artifact of using the false velocity is apparent, with the incorrect deeper location of the reflector depth under the low velocity blocks and the diffraction smiles. These smiles are occasionally interpreted as indicating over-migration with a velocity that is too high.

Since the most frequent objective of the application of prestack velocity analysis is to produce a velocity field for migration, migration will be used here as a quantitative measure of the accuracy of the velocity analysis.

\section{Reference Model}

The reference model used for the first ray tracing of the tomographic inversion is shown in Figure 4. It has a constant velocity and reflector artifacts that result from interpreting the velocity variations as reflector depth variations. The broad reflector slump under the left shallow velocity variation typifies the long wavelength result frequently observed from a statics solution.

The raypaths through this reference model contain some significant differences from those in the original model. The raypaths between a shot and the receivers for the original

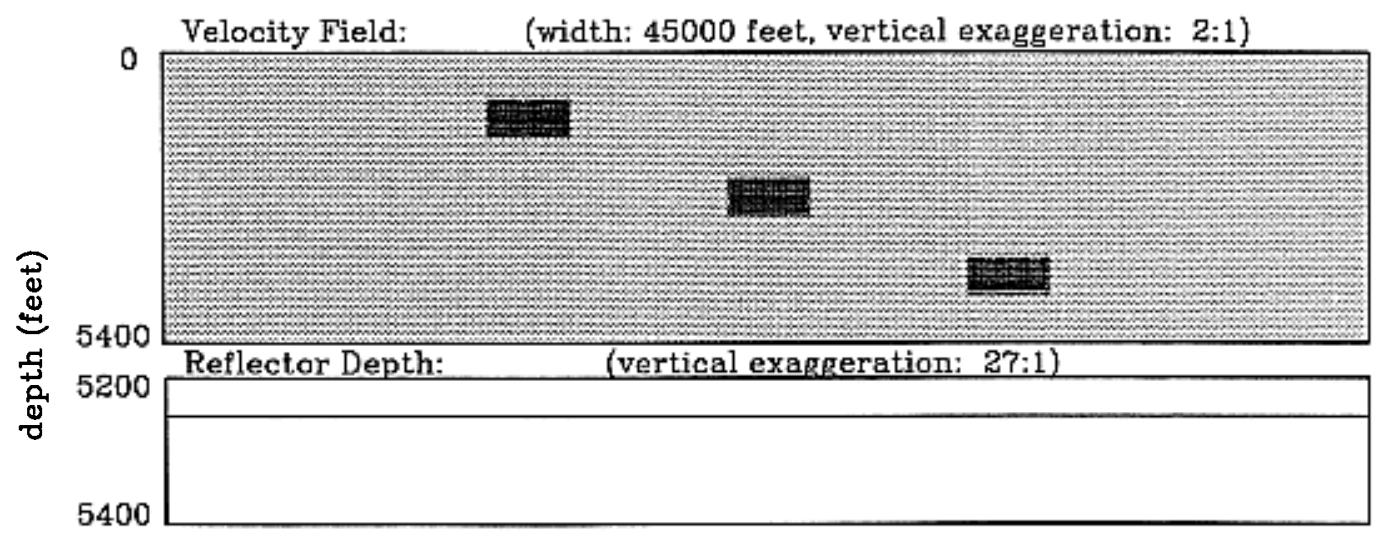

6000

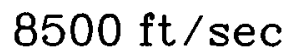

FIG. 1. Original model from which synthetic data are produced. The inversions attempt to reproduce this model. The velocity field contains three low velocity blocks $\left[V_{p}=6000 \mathrm{ft} / \mathrm{s}(1820 \mathrm{~m} / \mathrm{s})\right]$ in a background velocity of $8000 \mathrm{ft} / \mathrm{s}(2400 \mathrm{~m} / \mathrm{s})$. A flat reflector exists near the bottom of the model [at $5250 \mathrm{ft} / \mathrm{s}(1580 \mathrm{~m} / \mathrm{s})$ ]. The reflector is plotted on a separate scale so that vertical scale can be exaggerated. 


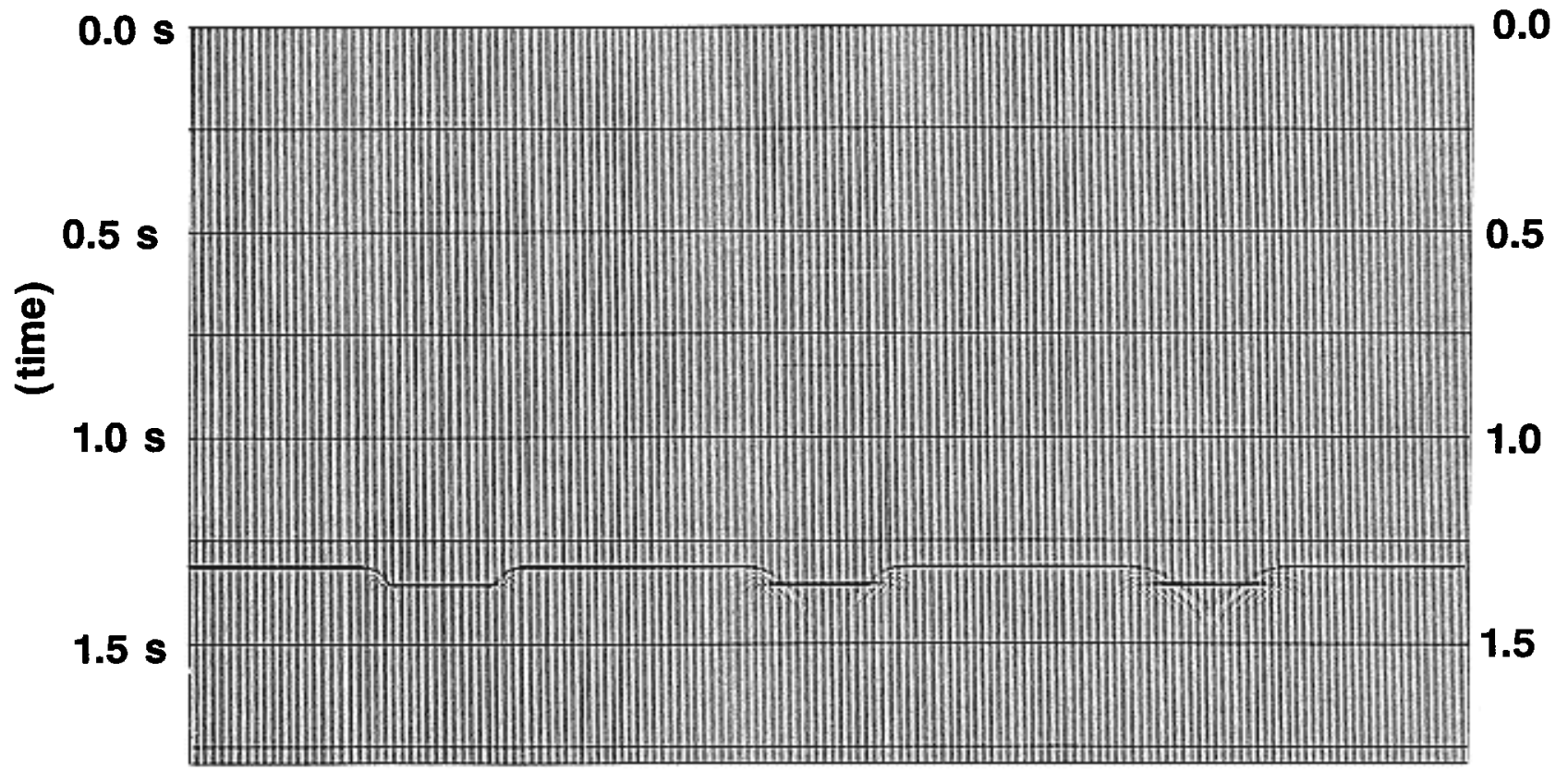

FIG. 2. Synthetic zero offset seismic section from original model of Figure 1. Data was produced using a 45 degree finite-difference one-way propagator. Larger diffractions exist under the right lower velocity variation and become smaller with the upper velocity variations.

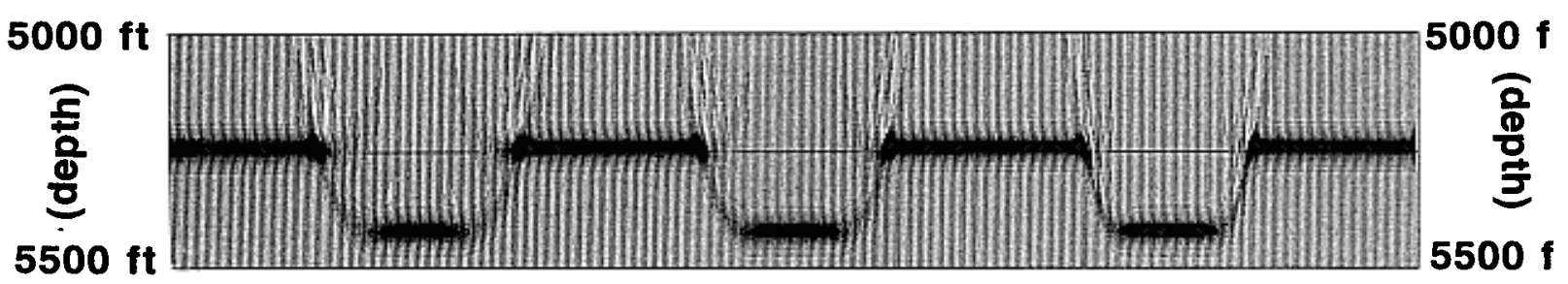

FIG. 3. Migration of zero offset section through the constant background velocity of $8000 \mathrm{ft} / \mathrm{s}(2400 \mathrm{~m} / \mathrm{s})$. The missing velocity variations have manifested themselves as false reflector depth variations. A larger migration smile exists under the upper left velocity variation. These migration smiles are sometimes incorrectly interpreted to result from a migration with an average velocity that was too high.

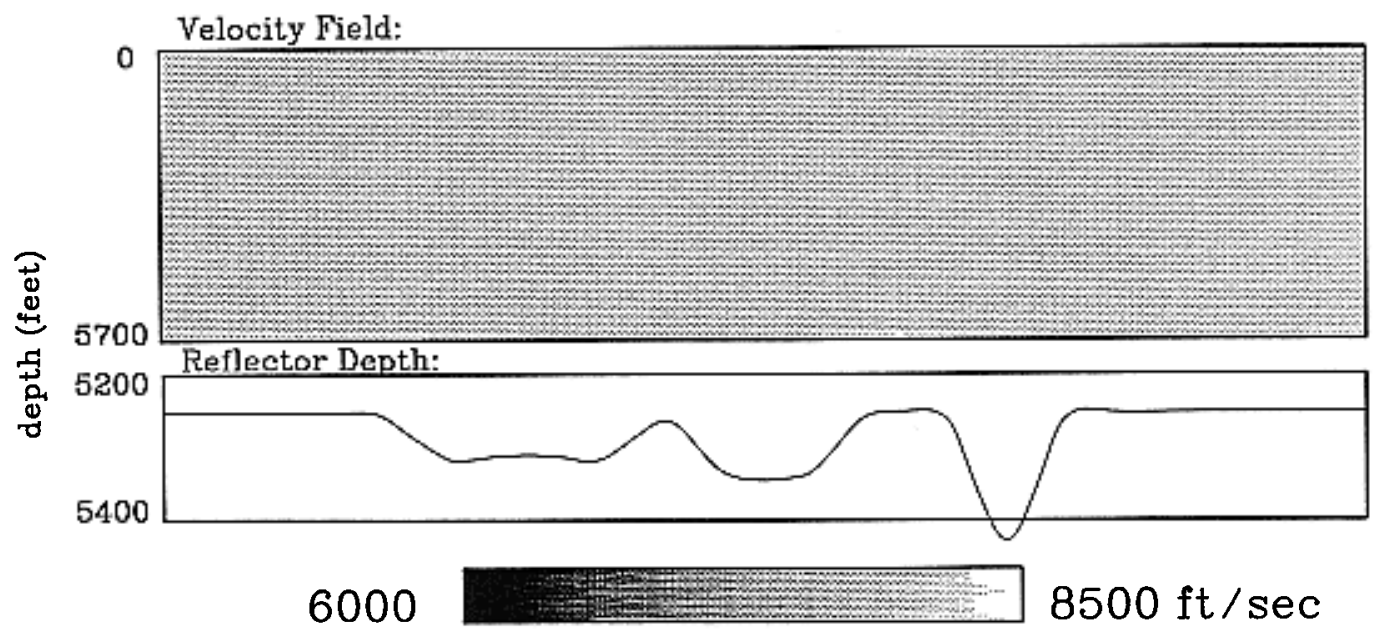

FIG. 4. Reference model used for ray tracing. Rays from this model are used to invert the traveltime data from the original model of Figure 1 . The reference model was chosen by assuming that the velocity variations were not resolved. The velocity of the model is a constant of $8000 \mathrm{ft} / \mathrm{s}(2400 \mathrm{~m} / \mathrm{s})$. 
model are shown in Figure 5a and for the reference model in Figure $5 b$.

Ideally, the tomographic inversion should adjust the reference model to look like the original model of Figure 1.

\section{Inversion of Model}

The inversion without constraints is shown in Figure 6a. The initial low velocity blocks have been smeared vertically and the reflector position depicts incorrect structure. The lowest velocity block is poorly inverted and causes severe reflector artifacts.

Figure 7a shows the migration of the zero offset data of Figure 2 through the inversion of Figure 6a. The reflector location in the migrated image closely follows that of the inversion except that diffraction smiles exist under the edges of the velocity variations.

\section{Retracing of Rays}

To improve on the raypath errors, new rays are traced through the inversion of Figure 6a. The inversion using these rays is shown in Figure $6 \mathrm{~b}$ and the migration through the inverted velocity field in Figure $7 \mathrm{~b}$. This migration shows the left and central side of the reflector to be more continuous than in the migration in Figure $7 a$. The artifacts at the right side of the model are not improved.

Figure $6 \mathrm{c}$ presents the result after two additional ray tracings and inversions. The amplitude of the two upper velocity variations continues to decrease slightly and the migration (Figure 7c) continues to heal the breaks in the refiector. In particular, the left side is nearly completely healed, although the diffractions are not entirely eliminated. The right side of the model shows no improvement. The additional ray tracings and inversions have not helped on this side of the model.

To emphasize some of the potential problems with the raypath errors, the inversion of Figure $6 c$ is repeated with little damping (i.e., a small value for $\varepsilon_{1}$ of Stork and Clayton, 1991) in Figure 6d. This inversion is the fourth one (after three loops of inversion and retracing of rays) yet significant raypath errors still exist for the right side of the model. In this case, the repeated retracing of rays and inversions appears to be unstable.

\section{Linear Inversion}

The artifacts remaining after the repeated ray tracings and inversions can be classified into two groups: nonlinear artifacts resulting from the raypath errors, and linear artifacts resulting from the inherent limitations of the seismic experiment. Examples of these inherent linear artifacts are the smearing from the limited angular ray coverage and the poor resolution between velocity and reflector depth. These features cannot be resolved even with the correct raypaths. The artifacts exist because models with and without the artifacts produce the same data.

It is not readily apparent which of the artifacts of the previous models are linear and which are nonlinear. To isolate the linear artifacts from the nonlinear ones, the mode] of Figure 1 is altered to contain lower amplitude velocity variations that do not significantly affect the raypaths. Figure $8 \mathrm{a}$ shows the new model used for inversion. The velocity variations are $\mathbf{4 0}$ times smaller than the model of Figure 1. This revised model will be used as the original model for all subsequent inversions. For these small amplitude velocity variations, the true raypaths are nearly straight. As before,

a)

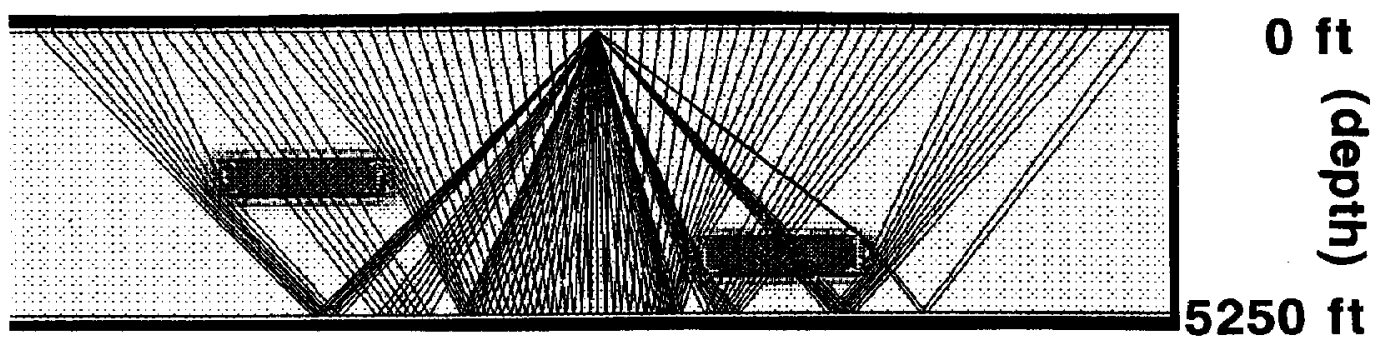

b)

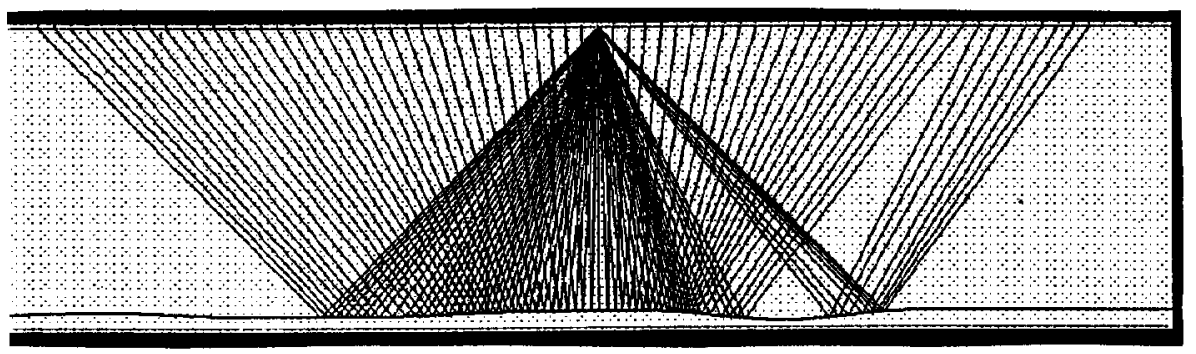

Fig. 5. (a) Raypaths through the original model showing how the velocity variations bend the rays. (b) Raypaths through the reference model showing how the reflector structure affect the rays. The rays of the reference ray are used to approximate the correct rays of the original model. The difference between the raypaths will introduce errors in the inversions. 
a)

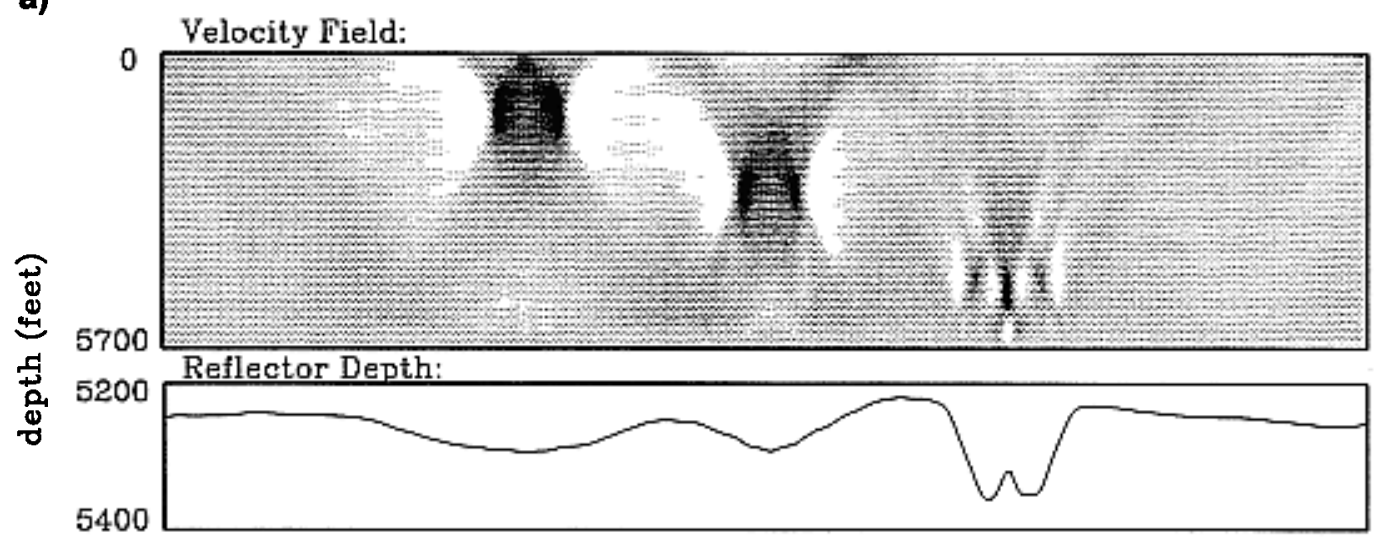

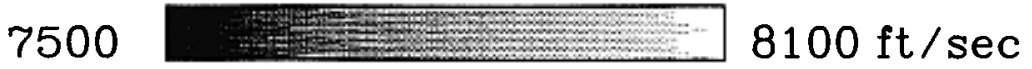

b)

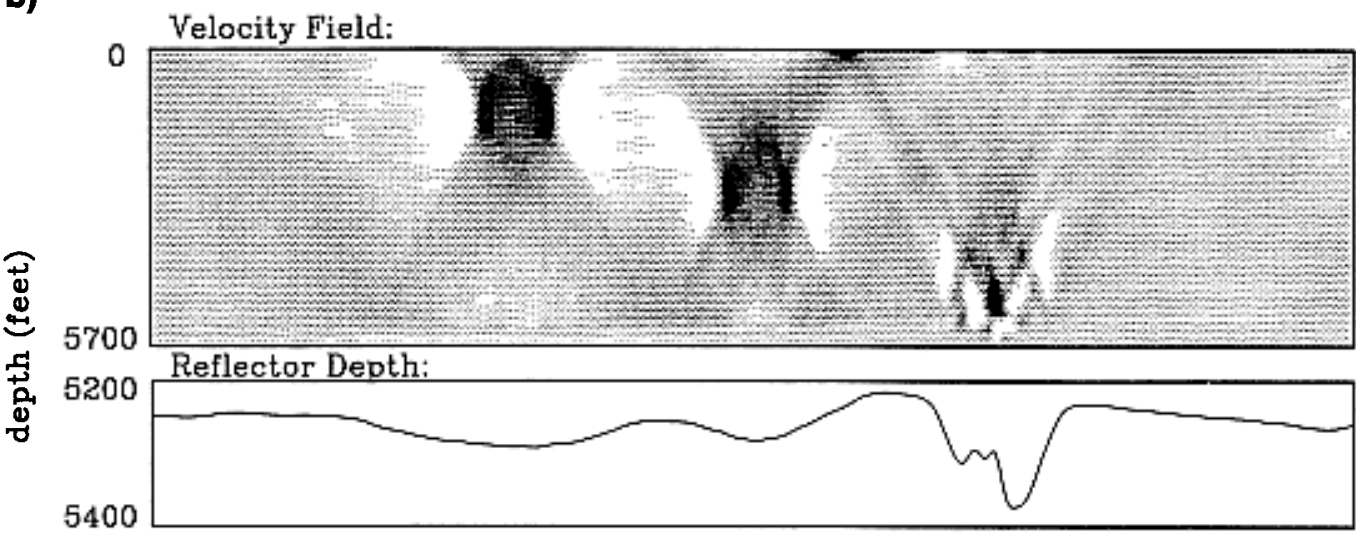

7500

$8100 \mathrm{ft} / \mathrm{sec}$

FiG. 6. (a) First inversion which attempts to correct the reference model of Figure 4 back to the original model of Figure 1. The amplitudes of the velocity variations are smaller (note scale change) and have been smeared vertically. The upper velocity variation is inverted best, while the lower one shows least improvement. The reflector structure is improved on the left side, but not on the right side. (b) Second inversion after retracing of rays through the previous inversion of Figure 6a. This retracing of rays is performed to improve on the errors introduced from using incorrect raypaths traced through the reference model of Figure 4 . No improvement is apparent, but the migration through this inversion (shown in Figure 7b) indicates significant improvement. (c) Fourth inversion after performing a total of two additional ray tracings and inversions over that of the previous inversion in (b). Inversion of velocity variations and reflector structure, particularly on the left side of the model, has been improved. Bizarre velocity variations and small scale false reflector structure are introduced on the right side of the model. (d) Inversion in (c) reproduced with little damping. The raypath errors that still exist after three inversions and retracing of rays have caused significant artifacts on the right side of the model. In this case, the repeated retracing of rays and inversions appears to be unstable. 
the reference model, shown in Figure $8 \mathrm{~b}$, has a constant background velocity and incorrect reflector structure caused by interpreting velocity variations as reflector depth variations. These reflector variations are also of such small amplitude that they will not affect the raypaths. The raypaths in the reference model will be nearly identical to those in the original model. The inversions, therefore, contain almost no nonlinear artifacts.

Figures $9 \mathrm{a}, \mathrm{b}$, and $\mathrm{c}$ show three inversions to different minimum eigenvalues. The minimum eigenvalue to which we are able to invert is dependent on the data quality. The first inversion to a minimum eigenvalue of 0.20 has raised the reflector structure and started to invert the velocity variations. The inversion of the upper velocity variation has the greatest amplitude while those of the lower ones have successively decreasing amplitudes.

Inversion to an eigenvalue of 0.04 has increased the amplitude of the velocity variations and has continued to raise the reflector so its average depth is close to the correct level. While the upper two inversions of the velocity blocks now appear similar, the lower one is still poorly resolved. Only its edges are resolved. Close inspection also reveals that the extent of the vertical smearing has been slightly reduced. From our experience with real data (Stork, 1988), an inversion to an eigenvalue of 0.04 is generally the smallest possible with the noise of typical seismic data. High quality seismic data may allow resolution to smaller eigenvalue.

Continuing the inversion to an eigenvalue of 0.01 has corrected the reflector depth on the left side of the model but not on the right side. The long wavelength aspects of the reflector structure appear resolvable while the medium and short wavelength aspects do not.

\section{SYNTHETIC MODELING WITH CONSTRAINTS}

To attempt to correct the problems encountered in the previous examples, a variety of constraints are imposed. They are: velocities derived from boreholes, limitations on the maximum or minimum velocities possible, restrictions on the location of the velocity variations, constant velocity zones, and a minimum entropy criterion to collapse vertical velocity smearing. While these constraints are applied here to solve linear problems (where no raypath errors exist), they can be equally applied to improve on nonlinear problems (where raypath errors exist).
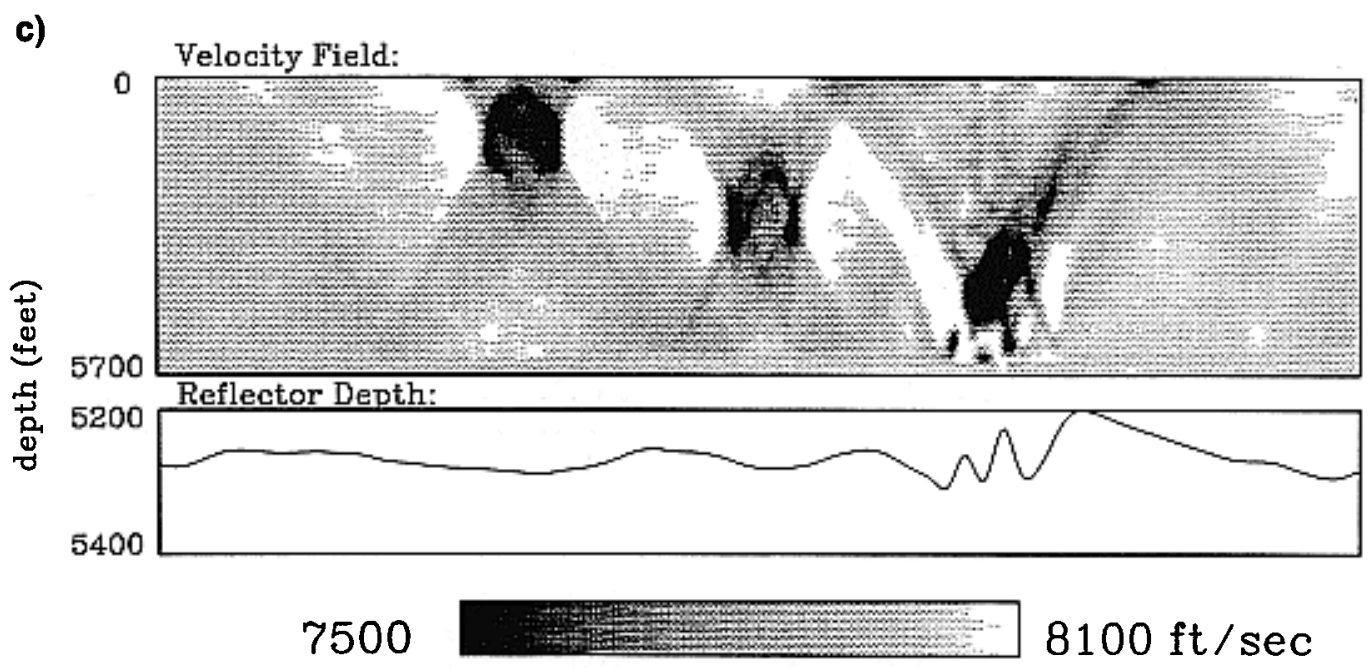

d)

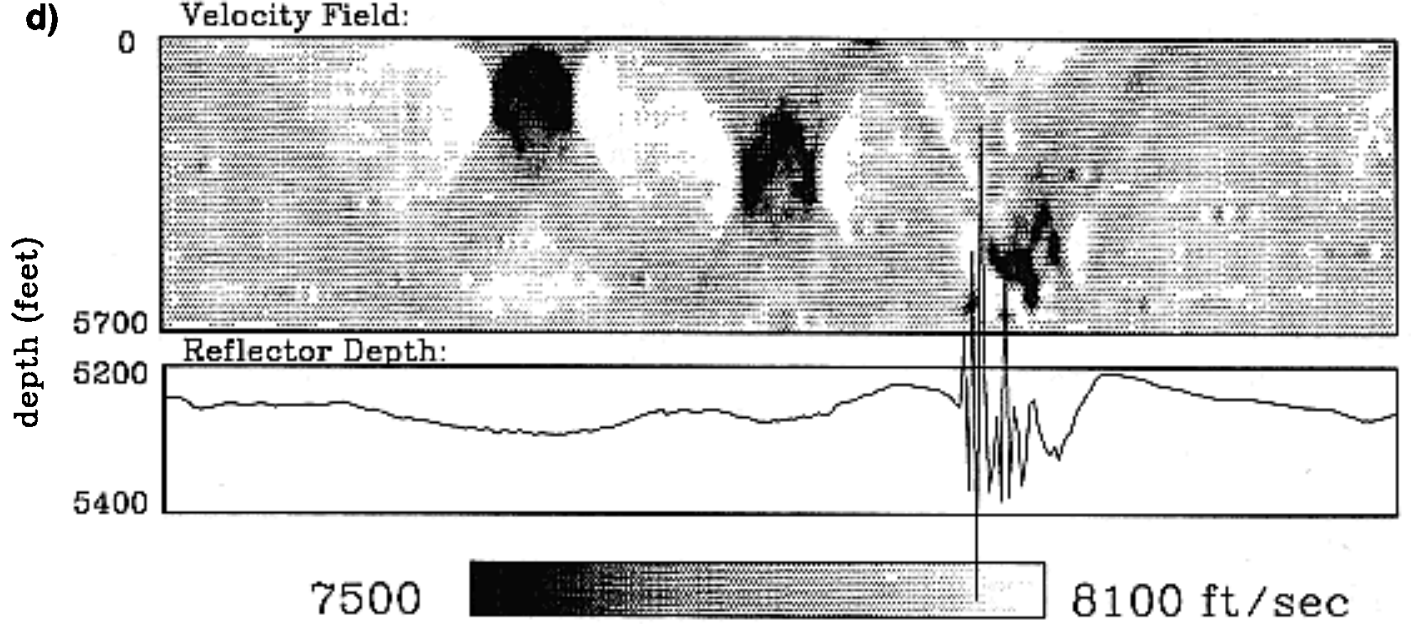

FIG. 6. (continued) 


\section{Borehole Velocity Constraints}

Velocity information (such as from check shots or sonic logs) from wells along a seismic line can be used to constrain the inversion. We implement a borehole velocity constraint by not allowing the velocity at the borehole to vary and damping variations in the vicinity of the borehole. The damping is gradually relaxed with increasing distance from the borehole, as described in Stork and Clayton (1991).

The inversion (to a minimum eigenvalue of 0.04 ) with borehole constraints is shown in Figure 10. The locations of the boreholes are plotted on the inversion. Comparison with the corresponding unconstrained inversion of Figure $9 b$ shows the reflector to be significantly improved on the left side of the model. However, underneath the right velocity variation, the reflector structure has not been well corrected even though the two boreholes exist near the velocity variation. Close inspection of the velocity field reveals that the velocity smearing has been reduced.

\section{Min-max Constraint}

The unconstrained inversion of Figure $9 \mathrm{~b}$ shows that there are areas of velocity increase even though the velocity variations of the original model are all negative. These areas of velocity increase appear as white. If, for instance, the velocity variations are known to be caused by gas and if geologic information indicates the velocity of the rock units unsaturated by gas is fairly uniform, we can expect the velocity not to increase beyond the unsaturated rock veloc- ity. Thus we impose the constraint to not allow velocity increases. We call this a min-max constraint because it limits the maximum or minimum values the velocity is allowed to take on. The result in Figure 11 shows that the reflector is nearly perfectly inverted on the left side of the model and the velocity variations exhibit much less vertical smearing. On the right side of the model, under the lower velocity variation, the reflector is not significantly improved.

\section{Restrictions on the Locations of Velocity Variations}

We apply a constraint that restricts the locations where the velocity is allowed to vary in the inversion of Figure 12. The boxes where velocity variations are allowed are approximately twice the width and $2 \frac{1}{2}$ times the height of the actual velocity variations of the original model. The inversion shows that the reflector is remarkably flat for the middle and left side of the model. The right shows marked improvement over the false reflector structure of the reference model.

This constraint is implemented by setting $\mathbf{F}_{2 i j}$ of Stork and Clayton (1991) to zero for the locations where the velocity is not allowed to vary. This constraint can also be implemented by setting the model weights $\left(\omega_{j}^{\mathrm{s}}\right)$ of Stork and Clayton, 1991) for the locations to zero.

\section{Constant Velocity Zones}

In some areas, one can assume certain lithologic units contain little or no velocity variations. For instance, gas a)

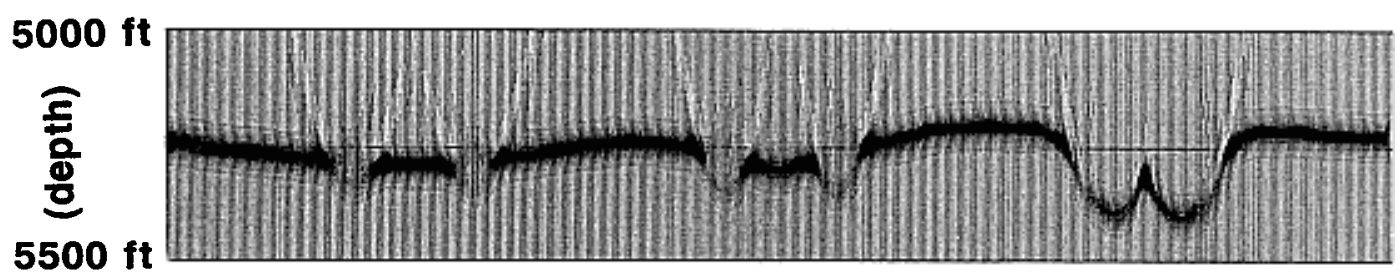

b)

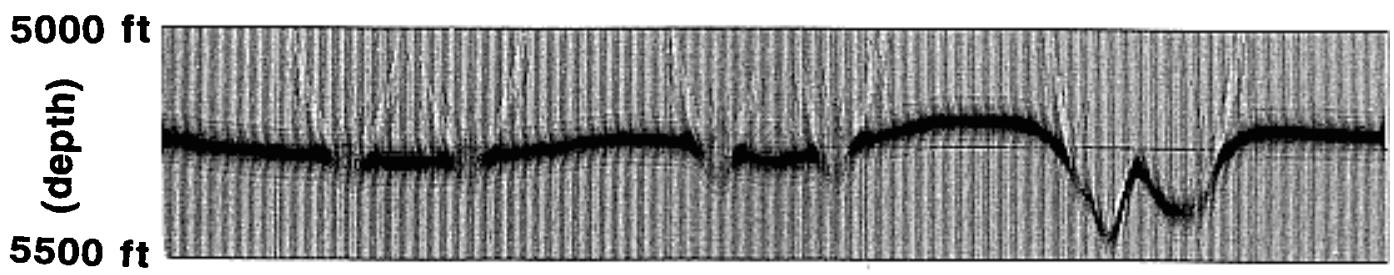

c)

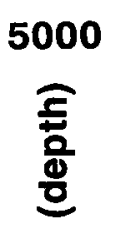

$5500 \mathrm{ft}$
$5000 \mathrm{ft}$

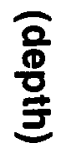

$5500 \mathrm{ft}$

$5000 \mathrm{ft}$

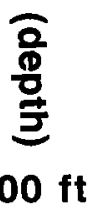

$5000 \mathrm{ft}$

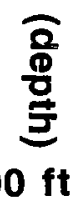

FIG. 7. Migrations of zero offset data of Figure 2 through the velocity inversions of the previous figures. (a) Migration through first inversion of Figure 6a. Migrated reflector position follows inverted reflector except that diffraction smiles have been introduced under the edges of the velocity variations. These smiles are sometimes interpreted as indicating over-migration of data. (b) Migration through second inversion of Figure $6 \mathrm{~b}$. The diffraction smiles on the left side of the previous migration are reduced. (c) Migration through final inversion of Figure $6 \mathrm{c}$. Diffractions are further collapsed on the left side. The vertical smearing has not significantly affected the focusing of the migration. The artifacts from raypath errors on the right side show little improvement. In this case, the repeated retracing of rays and inversions appears to be unstable. 
pockets may not exist in shale. In areas where velocity variations are caused by gas, known shale layers can be set to have a constant velocity. The inversion in Figure 13a has set certain depths to have a constant velocity. This constraint is implemented using the $\underline{F}_{1}$ filter as explained in Stork and Clayton (1991). This constraint has eliminated much of the vertical smearing, but accuracy of the reflector depths has not been improved.

\section{Min-max Constraint Combined with Velocity Variation Restrictions}

In the inversion with restricted velocity variations, the velocity in some areas has increased significantly. We repeat the inversion with the addition of the min-max constraints.
The result in Figure 13b shows most of the reflector artifacts to be removed, except again under the lower velocity variation on the right side. The reflector position from this inversion is very similar to that in Figure 11, which had only a maximum velocity limit.

\section{Minimum Entropy Constraint}

The minimum entropy constraint is a method of collapsing the vertical smearing of the limited angular ray coverage by favoring the concentration of velocity variations in a small area. It is implemented here with a penalty function in repeated inversion as described by Stork and Clayton (1991). The inversion in Figure 14 shows the constraint to be largely successful in collapsing the vertical smearing. This inversion was performed with the correct reflector depth fixed throughout the inversion.
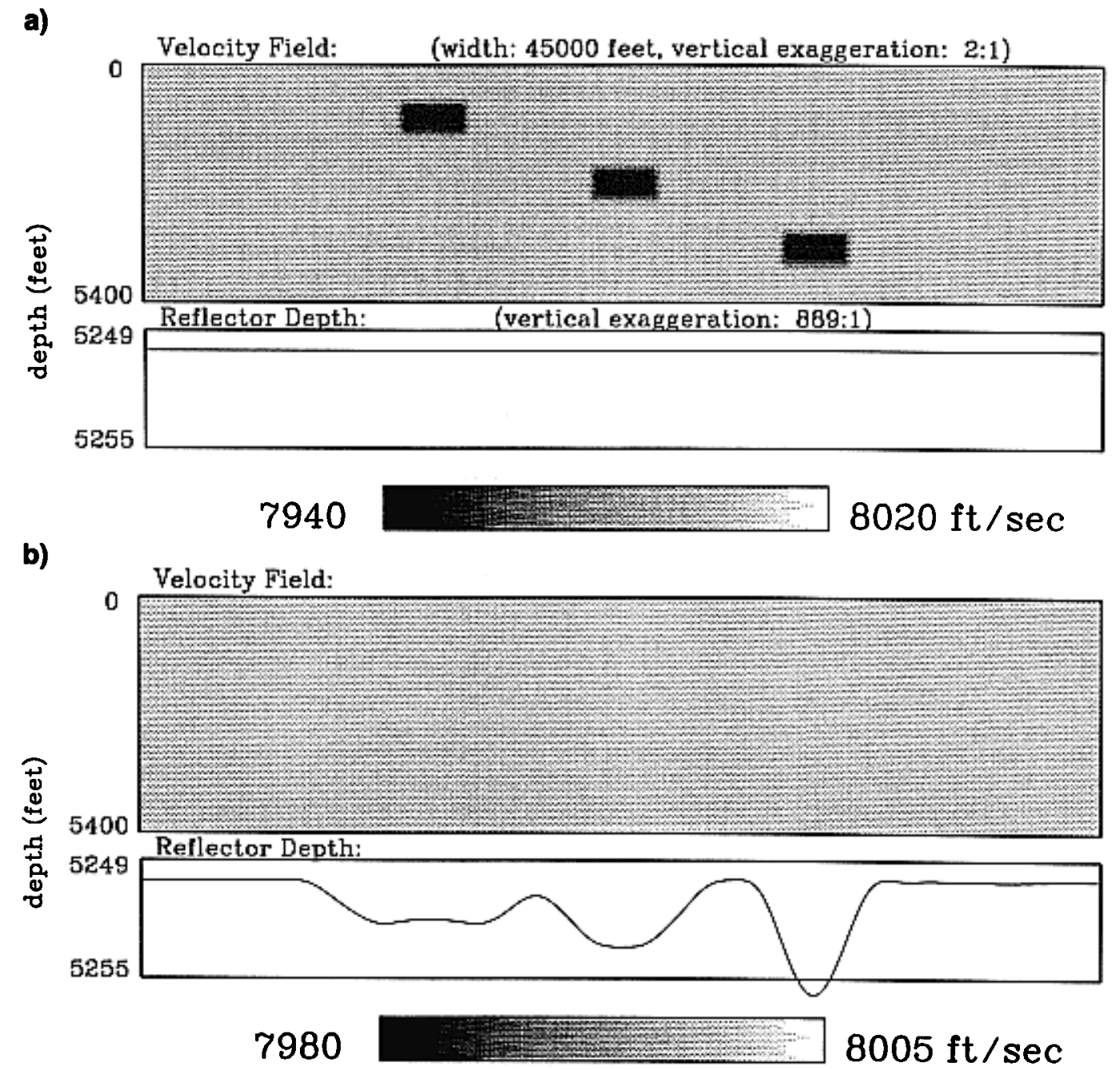

FIG. 8. Original model with low amplitude velocity variations and reference model for linear inversions. Although both models have the same structure as before, the amplitudes of the velocity variations and structure variations have been reduced by a factor of 40 . These small amplitude variations do not significantly effect the raypaths. Thus, the raypaths through each model are identical and the inversion contains no raypath errors (i.e., no nonlinearities). The inversion will contain only linear artifacts. (a) Original model with blocks of velocity $7950 \mathrm{ft} / \mathrm{s}(2400 \mathrm{~m} / \mathrm{s})$. (b) Reference model with velocity variations interpreted as structure variations. 
a)

Velocity Field:

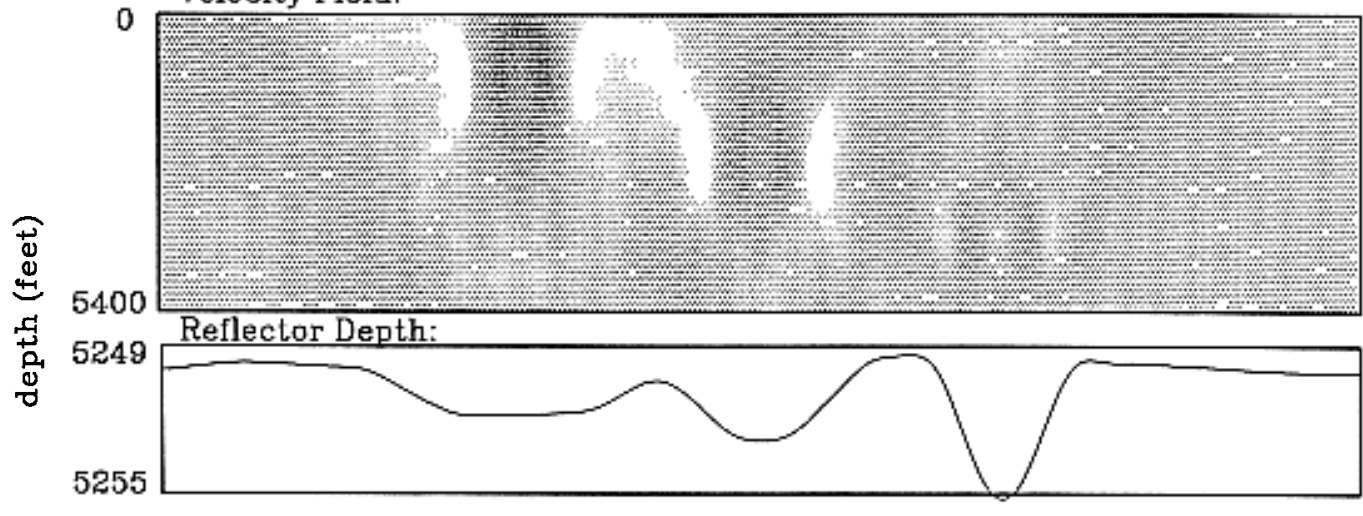

7980

$8005 \mathrm{ft} / \mathrm{sec}$

b)
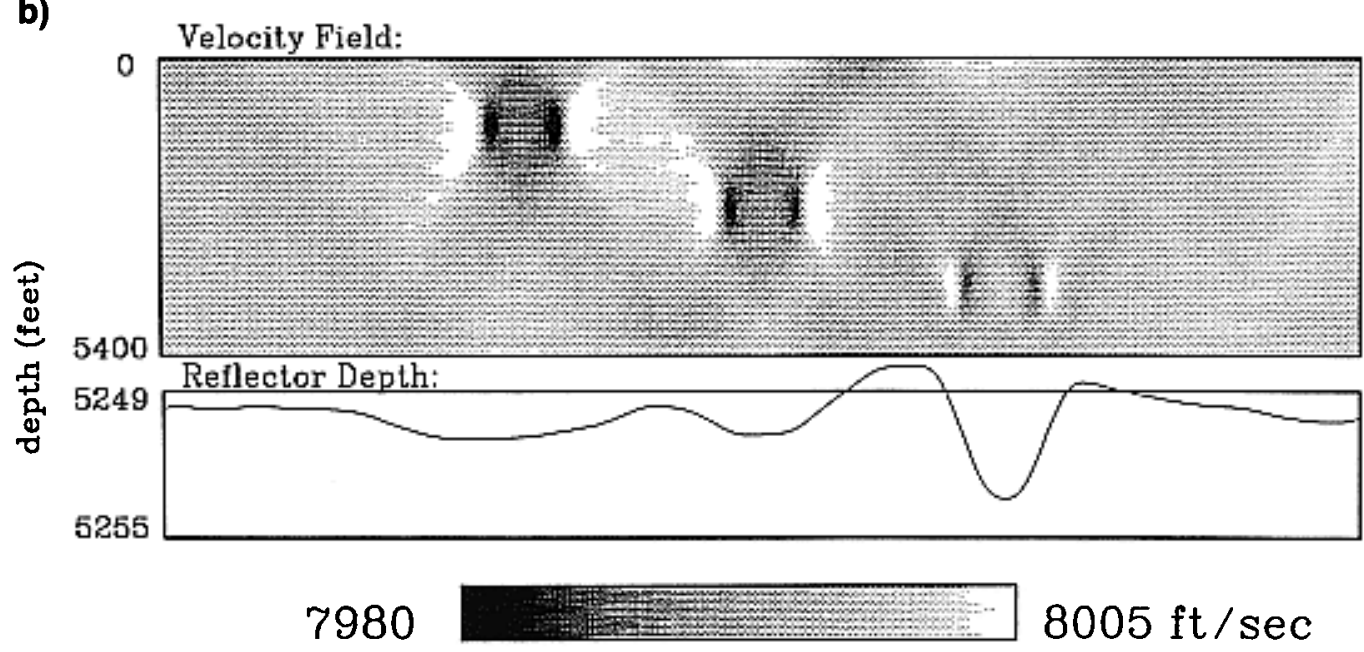

c)

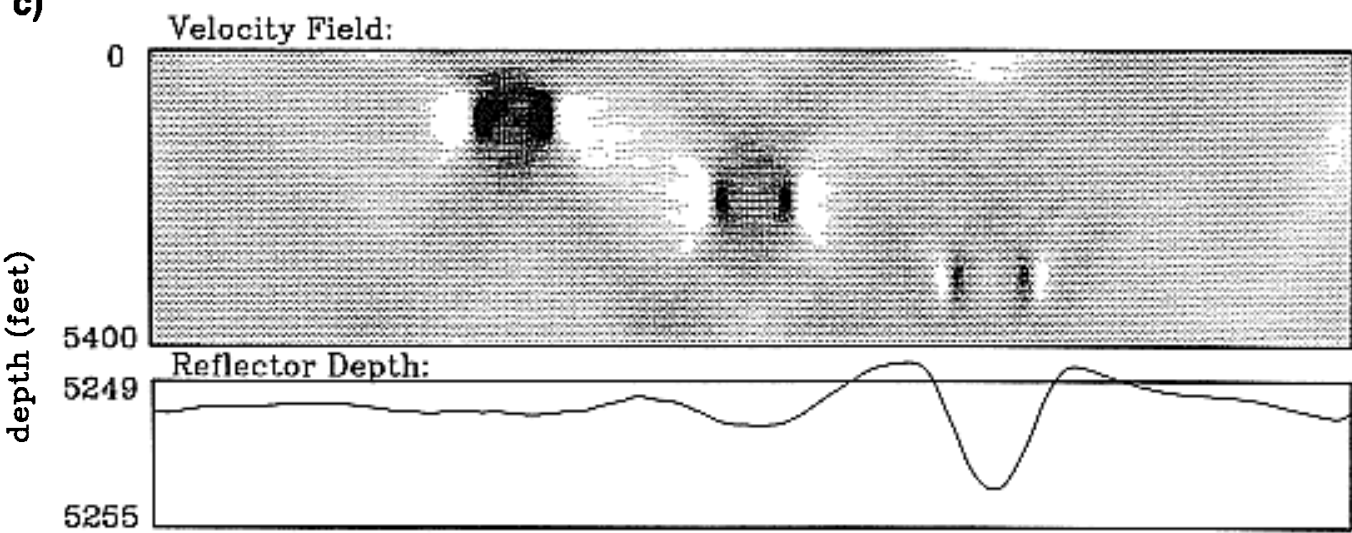

7980

$8005 \mathrm{ft} / \mathrm{sec}$

FIG. 9. Inversions without raypath errors to different minimum eigenvalues. (a) Linear inversion over eigenvalue range of 1.0-0.2. Velocity variations are not well resolved. Reflector structure has not been improved much. (b) Linear inversion over eigenvalue range of 1.0-0.04. Velocity variations and reflector structure on left side of the model are well resolved, but not those on the right side of the model. (c) Linear inversion over eigenvalue range of 1.0-0.01. Inversion is further improved on the left side but not on the right side. 


\section{DISCUSSION}

The problems demonstrated by the application of reflection tomography for velocity analysis can be grouped in three categories: smearing from the limited angular ray coverage, poor resolution between velocity variations and reflector depth variations, and nonlinear errors from ray tracing through a reference model.

\section{Vertical Smearing}

The vertical smearing of the structure from the limited angular ray coverage is the most noticeable artifact in a reflection tomographic inversion. Despite the smearing, the result is still quite useful since migration is not significantly compromised by this vertical smearing. The examples, such as Figure $7 \mathrm{c}$, show that migration through the smeared result produces a nearly correct image. The insensitivity of migration to the vertical smearing can be explained by viewing migration as a vertical integration process through the velocity.

However, it may be difficult to make geologic interpretations of a velocity field that is smeared vertically. Since geologic structure is mostly horizontal in many areas, the vertical smearing can significantly distort a velocity field. It is desirable to reduce the smearing.

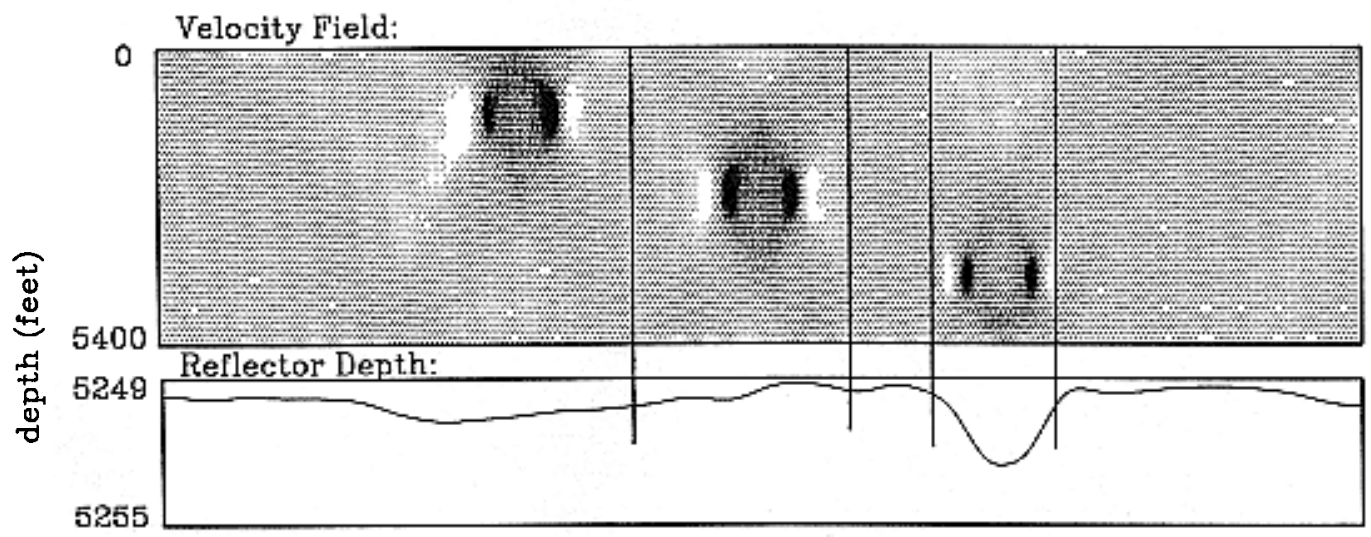

7980

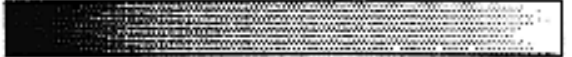

$8005 \mathrm{ft} / \mathrm{sec}$

FIG. 10. Linear inversion with borehole velocity constraints. The velocities and reflector depth within each borehole were fixed to the correct value. This inversion corresponds to that of Figure $9 \mathrm{~b}$ without constraints. Reflector structure is improved with the constraints, although not all artifacts have been removed. Note that the reflector pull upward between the two lower velocity variations is now removed.
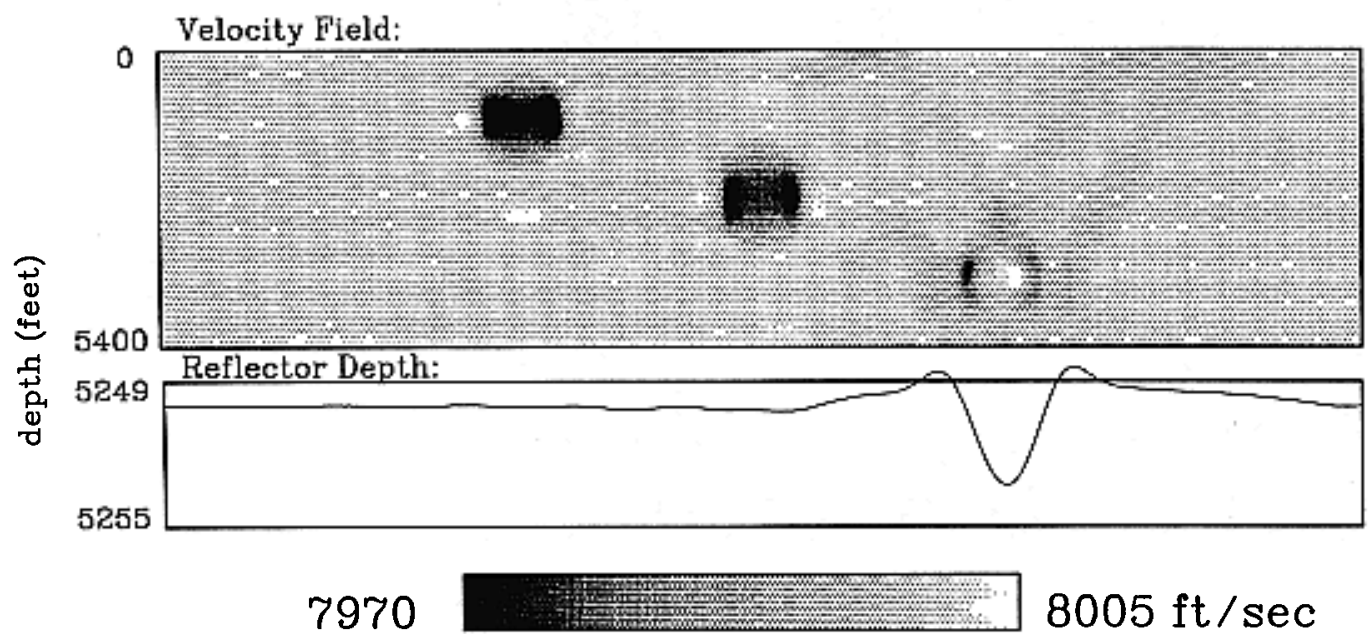

$8005 \mathrm{ft} / \mathrm{sec}$

FIG. 11. Repeated linear inversions with a maximum velocity limit. Inversion with the constraint that only allows the velocity to decrease but not to increase. This constraint is justified if it is known that velocity variations are only caused by gas and that the uncontaminated rock has a fairly uniform velocity. The reflector and velocity variations are very well resolved on the left side of model, but not on the right side. Note that the vertical smearing has been significantly reduced. 
Resolution between Velocity Variations and Reflector Depth Variations

The synthetic examples here show that unconstrained prestack velocity analysis does not always produce accurate reflector depths for the geometry used in these models. Incorrect reflector structure in the starting reference model is frequently not corrected. In some cases, false structure is introduced. Some of the false reflector structure-the gradual or long wavelength aspects-can be removed by inversion to small eigenvalue if the data quality warrants it. However, the medium and small scale refiector structures are not improved by inversion to small eigenvalue. In Figure $9 \mathrm{~b}$, the inversion to small eigenvalue has reproduced the correct average reflector depth but the smaller scale structure remains uncorrected. These examples demonstrate that a traveltime ambiguity exists between certain types of velocity variations and reflector depth variations. Apparently, velocity variations can mimic the hyperbolic moveout curve of one reflector at an incorrect depth. This observation is supported by the analytic results of Stork (1992). Moreover, our modeling analysis is performed with a cable length twice as long as is generally used in most seismic surveys which significantly improves resolution.

Santosa and Symes (1989) demonstrate that having data from more than one continuous reflector at different depths improve the resolution of the depths to all the reflectors. But with only one reflector, they too come to the conclusion that most components of the reflector depth are unresolvable. Although we have not studied the resolution of multiple reflectors, given the additional problems of 3D effects, anisotropy, edge effects, nonlinearities, and the variable nature of seismic data and geologic structures, we continue our discussion based on the assumption that in many cases the seismic traveltime problem contains significant ambiguity in resolving the depths to reflectors.

These two problems of velocity analysis (vertical smearing and velocity/reflector depth ambiguity) are not unique to our implementation of ray trace tomography. Traveltime data is insufficient to distinguish between models with or without these artifacts. Since the velocity variations associated with these artifacts are broad and smooth, they do not cause precritical reflections and their effect on the shape of the transmitted waveform is minimal; they mainly affect the traveltime and absolute amplitude of the transmitted waveform. We are unsure whether waveform methods can significantly improve on these artifacts. One indication of the insensitivity of waveform methods to these artifacts is that the migration through models with them is similar to those without them. Figure $7 \mathrm{c}$ shows that the migration through the smeared velocity field produces a flat reflector with few diffractions on the left side of the model.

\section{Nonlinear Errors}

Another major cause of artifacts are raypath errors resulting from inaccuracies of the reference model. The general approach to decrease these artifacts is to retrace rays through the previous inversion and to perform another inversion. This approach is shown by Farra and Madariaga (1988) and our results to work for certain artifacts, but not for others. Comparing the migrations in Figures $7 \mathrm{a}$ and $\mathrm{c}$, we see that the additional ray tracings and inversions have improved the focusing and depth locations of the reflector on the left side of the model. On the right side of the model, however, we see that additional ray tracings and inversions have not improved the reflector position. The middle of the model has improved marginally. In certain cases, such as with the use of small damping in Figure $6 \mathrm{~d}$, the inversion is unstable in the presence of reflector inaccuracies and actually introduces additional problems into the model. Farra and Madariaga (1988) find that the process of repeated ray tracing is much more stable for velocity errors of the reference model than it is for reflector location errors. The synthetic models here confirm their findings.

Our experience with many additional models, too numerous to present here, indicates that artifacts from incorrect raypaths are not predictable. We find they are highly depen-
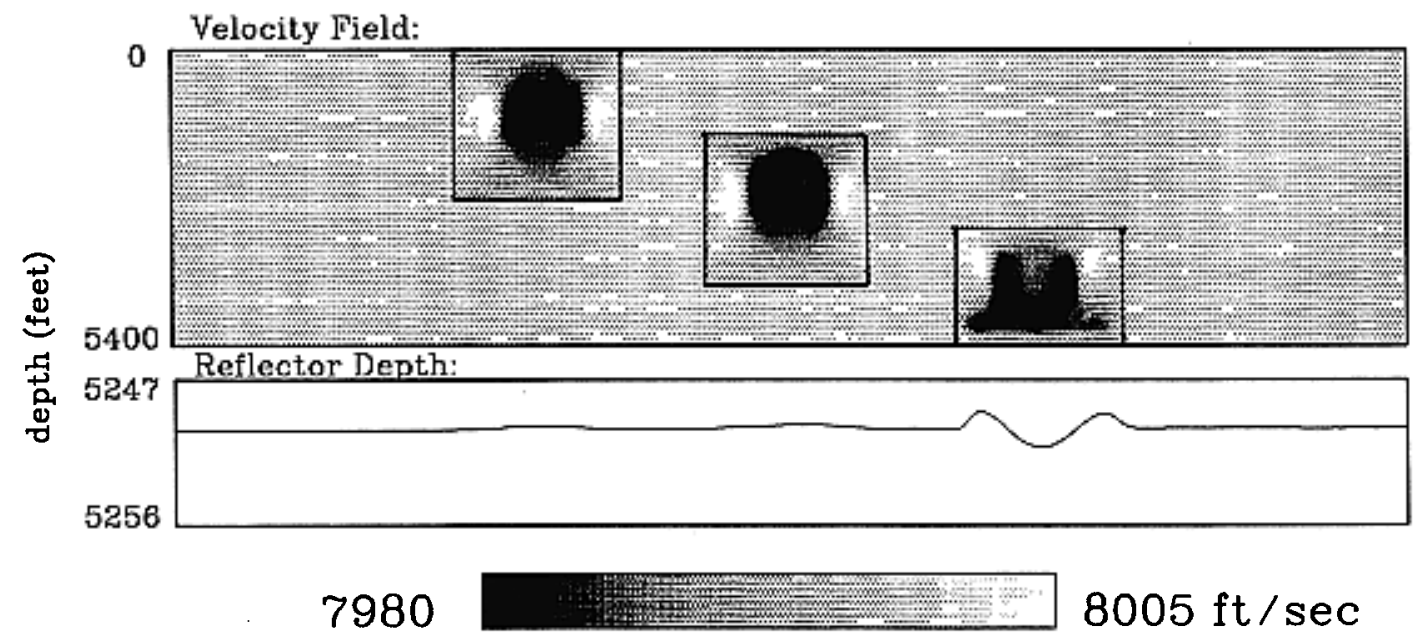

FIG. 12. Linear inversion with the constraint that restricts the location of the velocity variations. Only velocity within the marked boxes is allowed to vary. The reflector position of this inversion is well inverted on the left and middle side of the model and even the right side is significantly improved. 
dent on the original model and the reference model. We discourage the assumption that this process of the repeated application of the linear inversion (retracing of rays) will solve all or even most of the nonlinear artifacts (errors from tracing rays through a reference model).

A two-dimensional analogy of this nonlinear iteration process is like a blind man, able to jump long distances, trying to find the bottom of a valley. The direction and distance to jump is determined by the slope and curvature at his feet. Each jump represents one linear inversion. Since a linear inversion step is a nontrivial expense, we can only perform a few of these jumps relative to the total number of possible models. If the valley is smooth, the blind man will find the valley bottom quickly. But any intervening topography will send him off in the wrong direction. He may mistake a local depression for the bottom of the entire valley, or the topography may be so rough that he jumps endlessly around the valley almost randomly. While this analogy demonstrates some of the difficulties, it has only two dimensions and is relatively simple. Inversion for velocity analysis generally involves at least hundreds of dimensions and can be considerably more complex.

Nonlinear artifacts will still exist for other gradient inverse methods. Tarantola et al. (1990) draws on considerable experience to conclude that they also significantly impair an iterative application of Born wavefield inversion. This problem of nonlinear errors is a difficult one to address as it does not lend itself to theoretical analysis, and a modeling analysis is tedious and computationally expensive.

\section{Constraints}

With these three potential problems (smearing from the limited angular ray coverage, poor resolution between ve-
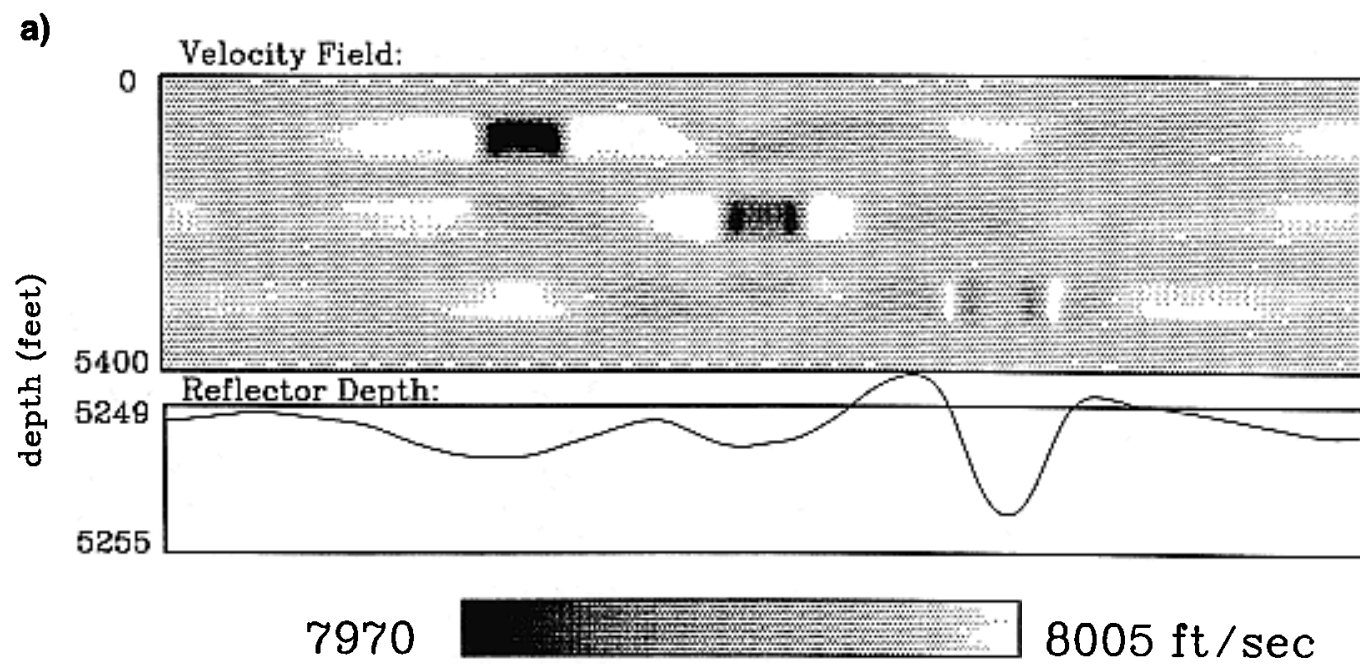

b)
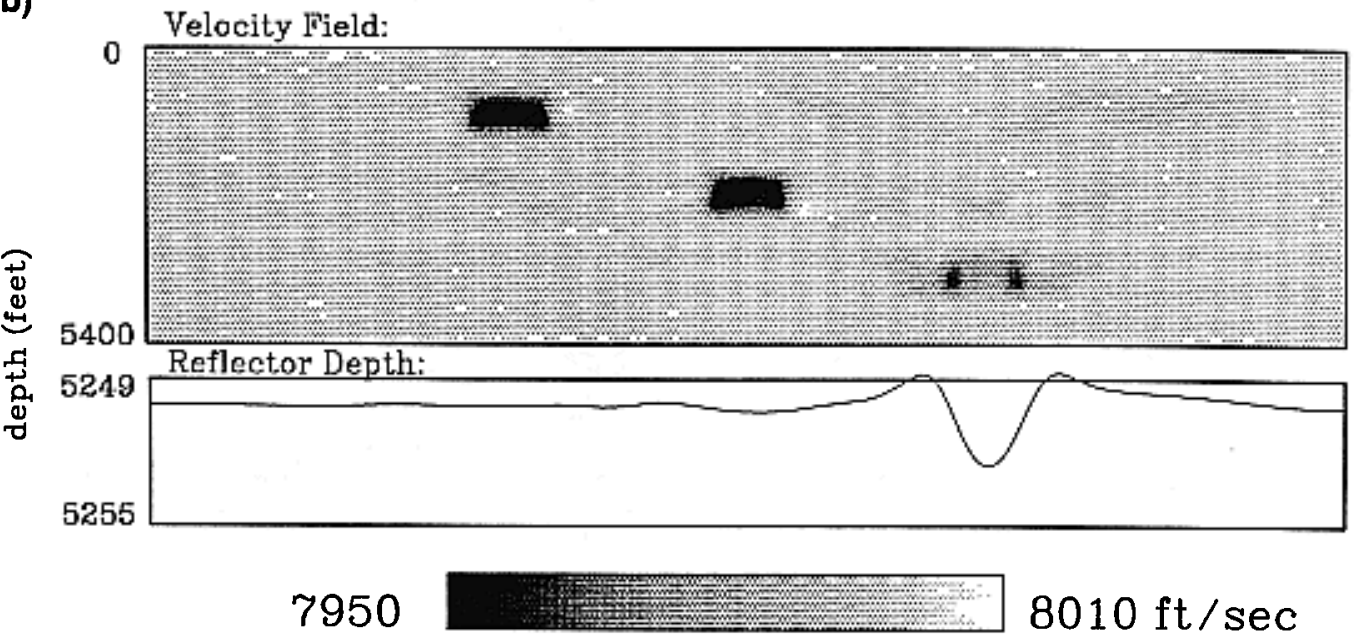

FIG. 13. (a) Linear inversion with constant velocity zones. Inversion with the constraint that only allows the velocity to vary at the correct depths. This constraint might be justified if shale units which do not contain gas-induced velocity variations are known to exist at certain depths. The inversion has not resolved the reflector structure very well. Note the strong positive velocity artifacts (white areas) that have been created. (b) Linear inversion with constant velocity zones and a maximum velocity limit. Inversion with a combination of the last constraints: velocity is only allowed to decrease and velocity variations are restricted to certain depths. The two left blocks are well resolved, but the right one still has its problems. 
locity variations and reflector depth variations, and nonlinear errors from the reference model) and the additional problems of edge effects, three dimensionality, anisotropy, and variable data quality, it is questionable whether general unconstrained prestack velocity analysis will produce a result that is useful. The result may be dominated by artifacts.

Fortunately, however, we have information at our disposal other than seismic data. Generally, much information is known about the nature of the structure and rocks in a given area when seismic data are collected. This geologic information can be used in the velocity analysis in the form of constraints to improve on the inherent problems of prestack velocity analysis.

The examples above demonstrate that numerous methods for constraining the velocity analysis are effective at resolving some of the problems of velocity analysis. The resolution of reflector depth is improved by incorporating borehole velocities, restricting the location of the velocity variations, and setting a maximum limit on velocity. The vertical smearing from the limited angular ray coverage is reduced by implementing a minimum entropy penalty function, constraining velocity variations to certain depths, and setting a maximum limit on the velocity. We are confident that these constraints can also be used to help reduce artifacts from reference model inaccuracies. Other possible constraints probably exist in addition to those presented here.

We foresee that there will be two types of approaches for using constraints to address the instability of generalized prestack velocity analysis: clear-cut and interpretational. The clear-cut approach is used when much is already known about the velocity variations. We just need a quantitative technique to take our data and produce a result as we envision it. In this case, velocity analysis has a well-defined objective and adequate constraints for stabilizing the velocity analysis are apparent before performing velocity analysis. For instance, in the Central Valley of California, it is generally clear when and where a gas pocket obscures a target below it. To properly migrate data through the gas pocket and to image below it, we need to accurately resolve the shape and absolute velocity of the gas pocket. In this case, we can constrain the velocity variations to occur only at the approximate known location of the gas pocket, and we can constrain the velocity to only decrease below the known velocity of the media when not saturated with gas. Other examples of well-defined velocity problems are permafrost variations in the North Slope of Alaska and Siberia, dynamic statics in the Saudi Arabian Peninsula, and fault blocks in the Idaho-Wyoming Overthrust Belt.

In these clear cut cases, where a well-defined objective for velocity analysis exists, the constraints serve to modify prestack velocity analysis to the unique characteristics of each application. We feel that given the variety of velocity problems, this flexible approach is more efficient in the long term than developing a new method for each application.

\section{Interpretational Constraints}

We anticipate that computer workstation tools can be developed that enable the geophysical interpreter to implement constraints interactively. The capability for flexible implementation of constraints by the interpreter would provide an interpretational approach for constraining prestack velocity analysis. This approach would be useful when sufficient constraints are not immediately apparent for adequately removing the inherent artifacts of generalized prestack velocity analysis.

As a result, the interpreter will have to consider a variety of constraints as he develops a velocity model that is both geologically reasonable and consistent with the data. The optimal constraints may only become clear after several repeated inversions. While the inversions may repeatedly have significant artifacts, such as can occur from raypath errors, an interpreter may recognize a trend and help guide

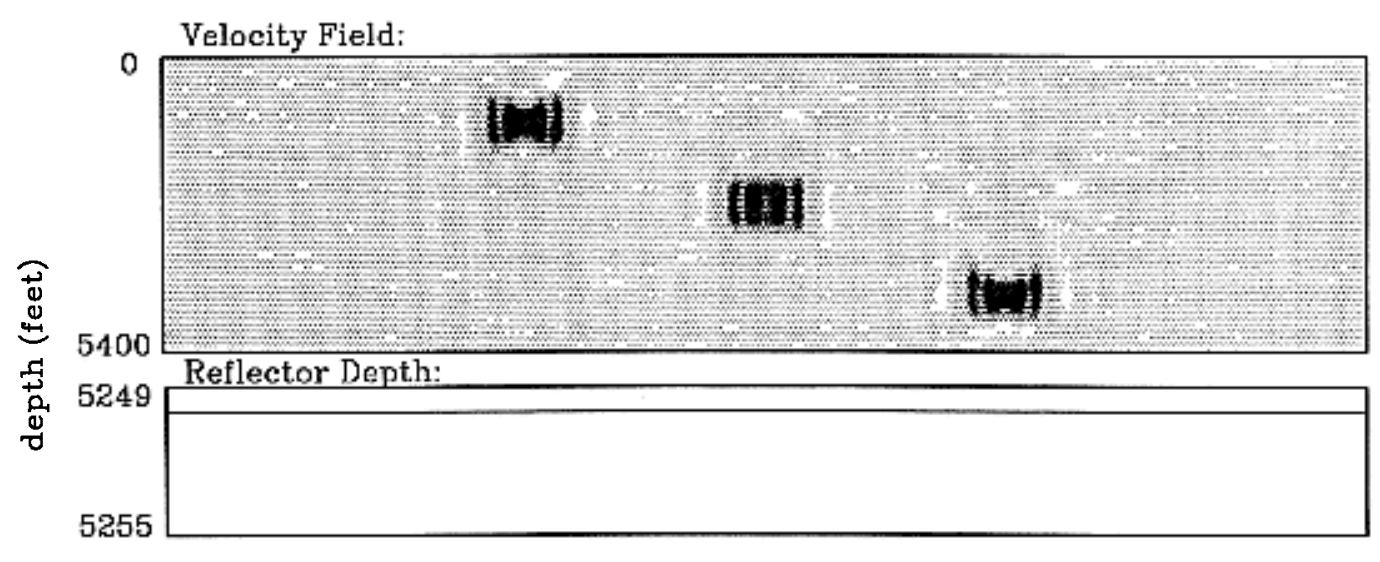

7960

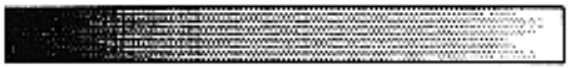

$8005 \mathrm{ft} / \mathrm{sec}$

FIG. 14. Repeated linear inversions with a minimum entropy constraint. Such a constraint attempts to collapse velocity variations so that they occur in the smallest possible area. In this case, it has successfully eliminated the vertical smearing. The reflector was fixed at the correct depth for this inversion. 
the process to some likely possibilities. In some cases, an interpreter may want to investigate several significantly different geologic possibilities.

In making decisions based on a data processing result, it is useful to know the accuracy of that result and the processing assumptions on which the result is based. In most situations, the final model from the velocity analysis will not be entirely satisfactory, and there will be other models that are nearly equally satisfactory. By using different constraints, an explorationist can explore the range of these other nearly satisfactory models. The use of an inverse approach allows many models to be considered rapidly. The explicit use of constraints helps clarify the assumptions on which a result is based. The use of inversion theory produces a quantitative measure of the sensitivity of the result on the data for a given set of assumptions.

\section{CONCLUSION}

Achieving a high-resolution velocity analysis is elusive with the use of seismic traveltime data alone. A prestack formulation cannot unambiguously resolve the velocity field. Generalized velocity analysis has a host of problems that are not specific to a certain inversion approach: the ambiguity between velocity and reflector depth, artifacts from reference model inaccuracies, and the velocity distortion from the limited ray angles available, among others.

These are inherent problems that can only be addressed by incorporating information other than seismic traveltime into the velocity analysis. The examples presented here indicate that some of these inherent problems can be improved with the implementation of relatively straightforward geologic constraints, such as well log information, constant velocity units, restrictions on the locations of the velocity variations, bounds on the velocities, and minimum entropy. These constraints and others provide the potential for producing an accurate interval velocity model for migration in areas of laterally varying velocity.

Using a general formulation for the velocity analysis with many optional inversion constraints provides much flexibility for the user. This flexible approach allows the user to modify the method to a unique application and to consider a wide range of possibilities as he reconciles data with geologic considerations. The prestack velocity analysis approach with flexible constraints can be thought of as an iterative, interpretational process that the explorationist can use to build a model that is both consistent with the prestack data and geologically reasonable.

The development of an accurate velocity model is important enough for seismic exploration to warrant the use of an interpretational velocity analysis approach by the explorationist.

\section{ACKNOWLEDGMENTS}

This work summarizes some of my (Christof Stork) experience with thesis research. I appreciate the inspiration, wisdom, and support of my co-author while pursuing this work, which proved to be more complicated than was expected at the outset.
Numerous other people (Biondo Biondi, Ken Bube, James DiSeana, John Etgen, Paul Fowler, Sam Gray, Jean Luc Guiziou, Gene Humphreys, Bob Langan, Ronan Lebras, Larry Lines, John Louie, Peter Mora, Chuck Moser, Fabio Rocca, John Scales, Allan Skorpen, Jay Thorseth, John Toldi, Jos van Trier, Sven Treitel, John Vidale, Marty Williams, Dan Whitmore, and Marta Woodward among others) have my gratitude for their insightful comments. Some aspects of this work were performed during my post-doctoral position at the Stanford Exploration Project.

This research was funded by a grant from Amoco and Robert W. Clayton's Presidential Young Investigators award. I thank Amoco, Arco, and Chevron for allowing me to pursue this work while employed with them for the summer. My exposure to data and seismic exploration through these companies were essential to this work.

My wife Terri was instrumental in enabling me to finish this paper.

This paper is contribution no. 4868 of the Department of Geological Sciences of the California Institute of Technology.

\section{REFERENCES}

Bishop, T. N., Bube, K. P., Cutler, R. T., Langan, R. T., Love, P. L., Resnick, J. R., Shuey, R. T., Spindler, D. A., and Wyld, H. W., 1984, Tomographic determination of velocity and depth in laterally varying media: Geophysics, 50, 903-923.

Bording, R. P., Gersztenkorn, A., Lines, L. R., Scales, J. A., and Treitel, S., 1987, Applications of seismic travel time tomography: Geophys. J, Roy. Astr. Soc., 90:2, 285-304.

Bube, K. P., Jovanovich, D. B., Langan, R. T., Resnick, J. R., Shuey, R. T., and Spindler, D. A., 1985, Well determined and poorly determined features in seismic tomography, Part II: 55th Ann. Internat. Mtg., Soc. Expl. Geophys., Expanded Abstracts, 608-610.

Bube, K. P., Langan, R. T., and Resnick, J. R., 1989, Unique determination of reflector depths in seismic reflection tomography: 59th Ann. Internat. Mtg., Soc. Expl. Geophys., Expanded Abstract, 918-921.

Deregowski, S. M., 1990, Common-offset migrations and velocity analysis, First Break, 8(6), 225-234.

Dines, K. A., and Lytle, R. J., 1979, Computerized geophysical tomography: Proc. IEEE, 67, 1065-1073.

Etgen, J., 1990, Residual prestack migration and interval velocity estimation: Ph.D. thesis, Stanford University.

Farra, V., and Madariaga, R., 1988, Non-linear reflection tomography, Geophys. J., 95, 135-147.

Faye, J. P., and Jeannot, J. P., 1986, Prestack migration velocities from focusing depth analysis, 56th Ann. Internat. Mtg., Soc. Expl. Geophys, Expanded Abstracts, 438-440.

Fowler, P., 1988, Seismic velocity estimation using prestak time migration: Ph.D. thesis, Stanford University.

Gardner, G. H. F., French, W. S., and Matzuk,.T., 1974, Elements of migration and velocity analysis: Geophysics, 39, 811-825.

Julien, P., Vujasinovic, Y., and Raoult, J. J., 1988, Depth continuous velocity analysis based on prestack migration, 58th Ann. Internat. Mtg., Soc. Expl. Geophys., Expanded Abstracts, 437441 .

Kennett, B. L. N, Sambridge, M. S., and Williamson, P. R., 1988, Subspace methods for large inverse problems with multiple parameter classes, Geophys. J., 94, 237-247.

Langan, R.T., Lerche, I., and Cutler, R.T., 1984, Tracing of rays through an heterogeneous medium: An accurate and efficient procedure: Geophysics, 50, 1456-1465.

MacBain, J., 1989, Interval velocity analysis for prestack depth migration, 59th Ann. Internat. Mtg., Soc. Expl. Geophys., Expanded Abstracts, 1233-1237.

Mora, P., 1987, Nonlinear two-dimensional elastic inversion of multioffset seismic data, Geophysics, 52, 1211-1228.

Santosa, F., and Symes, W. W., 1989, An analysis of least-squares velocity inversion: Soc. Expl. Geophys. geophysical monograph.

Sherwood, J. W. C., Chen, K. C., and Wood, M., 1986, Depths and interval velocities from seismic reflection data for low relief structures: Offshore Technology Conference Paper, 5161. 
Sneider, R., Xie, M. Y., Tarantola, A., 1988, Retrieving both the impedance variations and background velocity in reflection seismics using least-squares waveform inversion: 58th Ann. Internat. Mtg., Soc. Expl. Geophys., Expanded Abstracts, 10701072.

Stork, C., 1988, Ray trace tomographic velocity analysis of surface seismic reflection data, Ph.D. thesis, Calif. Institute of Technology.

1992, Singular value decomposition of the reflection seismology raypath inverse problem, Part 2: High-resolution analysis of a generic model: Geophysics, accepted for publication.

Stork, C., and Clayton, R. W., 1991, Linear aspects of tomographic velocity analysis: Geophysics, 56, 483-495.

Sword, C., 1987, Tomographic determination of interval velocities from reflection seismic data: The method of controlled directional reception: Ph.D. thesis, Stanford University.

Tarantola, A., 1984, Inversion of seismic reflection data in the acoustic approximation: Geophysics, 49, 1259-1266.
Tarantola, A., Crase, E., Jerris, M., Kohen, Z., Lindgren, J., Mosegaard, K., and Noble, M., 1990, Nonlinear inversion of seismograms: State of the Art: Presented at the 60th Ann. Internat. Mtg., Soc. Expl. Geophys

Thorson, J. R., 1984, Velocity stack and slant stack inversion methods, Ph.D. thesis, Stanford University.

van Trier, J., 1990, Tomographic determination of structural velocities from depth-migrated seismic data: Ph.D. thesis, Stanford University.

verWest, B., 1990, Subsalt Imaging using prestack depth migration and focusing analysis, Presented at 60 th Ann. Internat. Mtg., Soc. Expl. Geophys.

Williamson, P. R., 1986, Tomographic inversion of travel time data in reflection seismology, $\mathrm{Ph} . \mathrm{D}$. thesis, University of Cambridge. 1990. Tomographic Inversion in Reflection Seismology, Geophys. J., 100, 255-274.

Yilmaz, $O$., and Chambers, R., 1984, Migration velocity analysis by wavefield extrapolation: Geophysics, 49, 1664-1674. 